\title{
The effects of quality finishing surface on sealing and gluing
}

\author{
Alexandra Petrisor*, Gheorghe Nagit and Lucian Tabacaru
}

Gheorghe Asachi Technical University of Iasi, Department of Machine Manufacturing Technology, Romania

\begin{abstract}
In the assembly processes of mechanical parts from semifinished parts and finished parts there are different levels of roughness and different types of defects. This is mainly affecting the final surface of the finished parts. The founded defects can be from different categories like: irregular shape and surface, deformed edge, processing defects, little particles that combined with high pressures can make the parts crack. In the end this affects the sealing and the tightness of the assembled goods. After assembly it can also influence the performance of the resistance, the cycle life of the parts, the dullness and also the precision of final power packs. In this paper it is going to be established what are the parameters and the conditions for a stable sealing process that could survive to different environmental pressures. This results are presented after observation of various mechanical assemblies and their guidelines in which were determined the circumstance for a proper sealing. The main functions of the adhesion are the fixation and the sealing with a mechanical connection. In order to perform on maximum capacity this connection must be stable and strong and capable to hold to every impediment and test submitted. The following application used for gluing and sealing of electronic and mechanical components can be put it on single parts or in assembled parts and also for complex circuits, having a various applicability and utility in automotive industry.
\end{abstract}

\section{Introduction}

In the variety of assembly of different mechanical parts, all the way from the semi-finished parts to the final products, in which additional is including all the procedures, like machining, casting, forging, welding, they are distinctive levels of requirements of roughness and defects on the parts surfaces. [1] The defects of the part's surface can vary, from uneven covering, deformed edge even to casted products having defects of casting filling, chips, and very small cracks. The effect of this would be that on high tension submission, cracks can appear and destroy the final product. For this kind of weaknesses, if it will not be used a proper method of sealing, it will influence not only the quality and the lifetime of the products release and the automobile's production, but also the wear resistance by means of some mechanical influences, fatigue resistance, corrosion and last

${ }^{*}$ Corresponding author: dzetzit.alexandra@yahoo.com 
but not least the precision assembly of the pack. After this, it can also be affected performance of the whole group of parts in case a high current is applied, of the electrical cabinet, acceleration and braking in the safest conditions of an entire mechanical system, the increase of vibrations and environmental noise and the tightness loose. The real protective coverage against corrosion and also the final tightness tested and applied by different methods like gluing or welding is damaged only if from the beginning the proper parameters are not chosen.

One of the reason that is often met is that comes into view a surface that is not proper for a sealing due to tight collaboration between the material's microstructure, deformation during processing and physico-chemical reaction that happens in that time. The quick development and fast industrialization of scientific technology, in now a day's production it is been introduced for different machine on high scale, speedy and with high precision. Nevertheless, it is taken into account the flexibility, the integration on the final product, the autonomy, the intelligence and one of the main focus currently, on ecology. All the things mentioned above makes that in the end, the offer for the final product has a highly superior quality but it will not affect the geometrical characteristics of surface, the mechanical or materialistic proprieties.

The main duties of gluing are sealing and fixation of two or more components with a mechanical connection that will be stable and tight and it will be able to confront the different environmental factors. On the following points, the parameters and specification for this kind of association are mentioned.

The sealing's demands that are usually taken into account are adhesion of different material and finished surface, the resistance on higher temperatures, on change that are produced by this variations of temperature, the frequent attack of chemicals and vibrations. During the research are mentioned different concepts of assembly: the connection of the grooves, sealing surface and sealing by pressure (plastic or elastic).

In production, the gluing application is made in general through independent equipment or a system of automatic dosage, which are introduced in the final assembly's line, only after the finishing surface is previously prepared.

\section{Gluing}

The "gluing" term it is in general used for a way of fixation or a partial fixation for different electronic components (hybrid, plastic, metal). On the other side, the phrase of sealing is referring for assembly of two or more compounds mechanical stable for different electronics components. In mass production, this two processes are made by singular stations or fully automated which are integrated in the assembly system. (Figure1.)

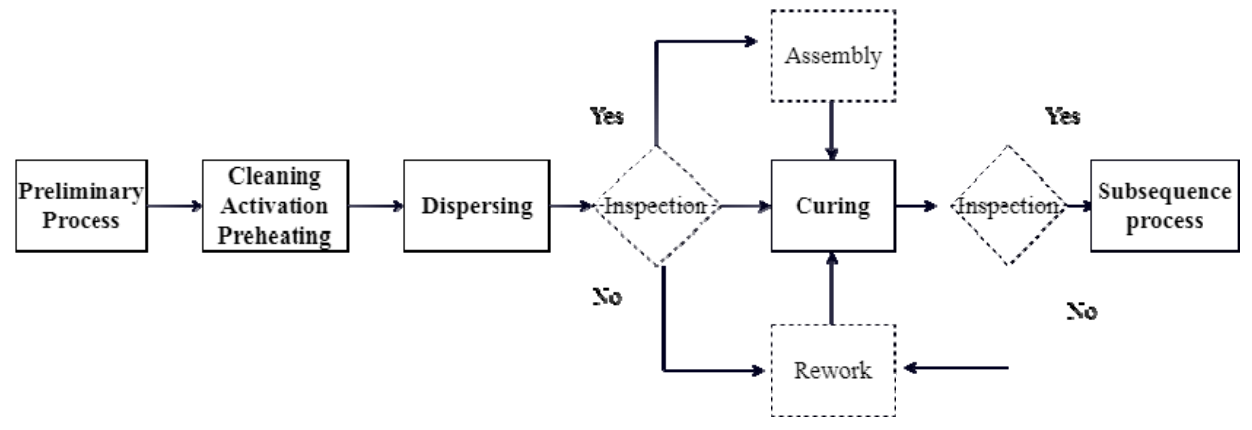

Fig. 1. Proposal for a subsequent processes for instance with CIPG (cure in place gasket). 


\subsection{Requirements for proper sealing and gluing}

In the following chapters are going to be presented some of the aspects that it should be taken in account when designing a part that will suffer a gluing process.

\subsubsection{Material's recommendation for usage}

The process of gluing in which the soldering paste is also introduced it is the most expended in the nowadays production. The quality of the material can influence in a big percentage the assembly process of the parts. [2] Assuring that the quality of the materials and the adhesives that are handled, especially their properties, will make the final product to work at fully capacity.

After identification of the various sealing materials on the basis of their tightness curves during this research, based on experiments, it had been determined three types of material: good for the gluing, medium material and low specification material for gluing. [3] The good types of material that good assure a safe operation: brass, zinc-plated finishes, polyamide (PA) - (Water absorption causes voids, drying is necessary) and polybuthylenterephtalate (PBT). From the category of "medium materials for gluing" are: die cast; aluminium, deep drawn components, gold-plated finishes, polycarbonate (PC) or containing blends, polyimide, polyphtalamide (PPA) - (Water absorption causes voids, drying is necessary), stainless steel, silver-plated finishes and tin-plated finish. Also the materials that should be avoided in this process are: liquid crystal polymer (LCP) activation of finish possible, polyphenylensulfide PPS - activation of finish possible, polyphenylensulfide PPS - activation of finish possible and teflon (PTFE) - not gluable [3].

Certain materials like chemicals, curing agents and plasticizers can inhibit the cleaning of the adhesive for soldering through the hardening of the additive. Ones of the most important additives used, tested without problems in production are:

$\checkmark$ Organotin and other organometallic compounds;

$\checkmark$ Silicone rubber containing organotin catalyst;

$\checkmark$ Sulfur, polysulphides, polysulphones or other types of sulfur components;

$\checkmark$ Amines, urethanes or amine-containing materials;

$\checkmark$ Unsaturated plasticizers with hydrocarbons;

$\checkmark$ Some solder flux residues.

If a substrate or a lower material it is questionable to the treatment of exfoliating, it is recommended that a compatibility test at low scale to be made into an application used. If a liquid presence or an untreated product is between the material and the gel, those in the end will be incompatibles [3].

\subsubsection{Surfaces and finishes}

The covering in which the soldering will be made for the projects was tested during several projects. After the research it has been detected that the following requirements are fulfilled, a lot of problems in the process and with the final parts will avoided. One of the items are cleanliness: until the freezing design of the final product, the cleanliness class for sealing, with the conformity of the standard 16939, with the following regulations must be part of the design of the parts:

- No contamination allowed

- No grease or oils allowed

- No agent used for injection or material casting

- The material temperature who will fix the joints

- Distribution of the unused material 
- Environmental conditions ( humidity, heat)

- The storage of the goods in production and other storages places that are affected by environment

All the surfaces need to be cleaned and degreased with naphtha, mineral spirits, methyl ethyl acetone or other adequate substance.

The surface's abrasion must be made easy and soft and is recommend it to be made every time possible due to the fact that is promoting a better clean area and by this, also the quality of the sealing will increase. Even the cleaning with acetone or IPA is util. Some cleaning methods can offer better solutions so that it should determine the best approach for the application.

The tension surface should have at least $38 \mathrm{mN} / \mathrm{m}$ and should be determined by using the ink test [1]. The polarity and nonpolarity of the material it must not be considered in this moment an impediment. The used surfaces must have a minimum adherence between $10 \mu \mathrm{m} \leq \mathrm{RZ} \leq 35 \mu \mathrm{m}$. The sealing material has a preponderant impact for all four pressure phases. This means that highlighted differences occur between the materials proved. Further important parameters are the space between the connection, the flange material and the temperature [3].

\subsection{General rules for designing the sealing surfaces}

In the following part, there are mentioned some aspects of designing points that were realized after several incidents with the products. (Figure 2.). One of the recommendations is that never to be used a 90 degrees angle. It should be specified that for 3rd parts the distribution of the channels, the flank angle must be 120 degrees.

The ventilation's holes from the casted parts needs to be assured, especially for every gluing area and one additional element will not be sufficient. This holes must be closed after the process of crimping the heads. The radius of any edges must be as big as possible for avoiding the infiltration of air and the bubbles resulting after this. The prevention of no big components and/or tall near the channel it is also important to avoid from the beginning.

Some of the aspects mentioned below can also be observed on the following diagram:

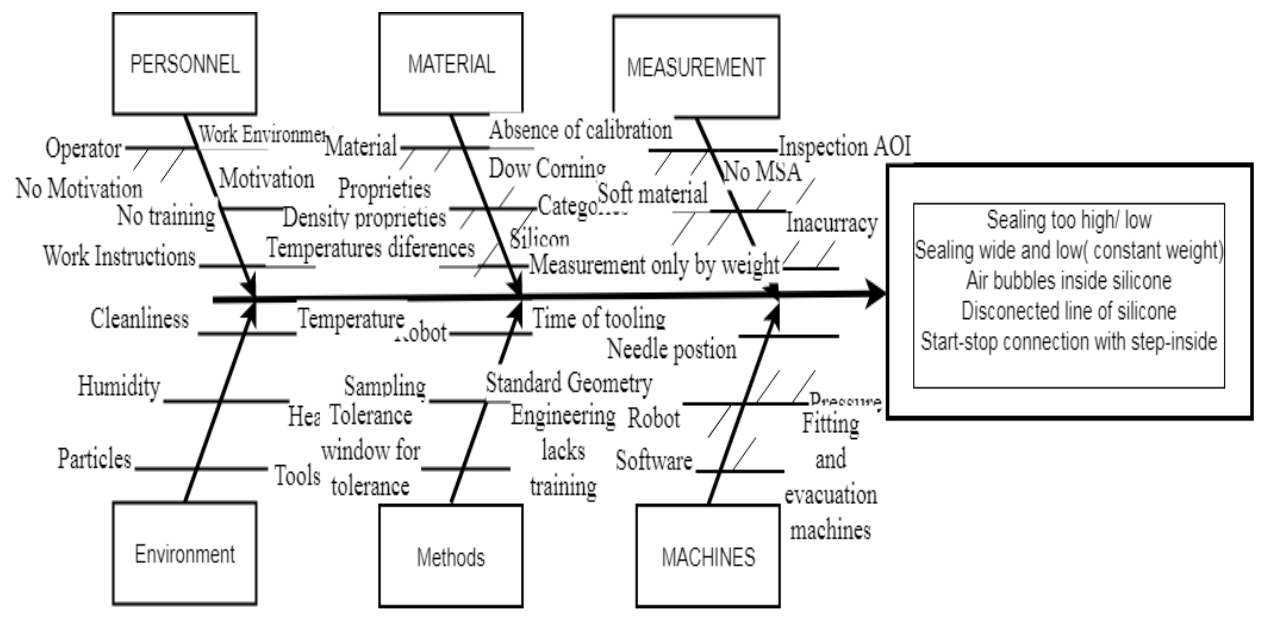

Fig. 2. Ishikawa $=$ factors for a proper sealing. 


\subsection{Recommendations for different assemblies' types}

\subsubsection{Type of assembly groove-tongue}

If we are speaking about curves and straight lines, every geometry is different. The minimum radius between the groove and the tongue it should be minimum $2,5 \mathrm{~mm}$. There are two different types of assembly: before and after the adhesive is spread. For having a proper gluing, some of the advices enunciated below should be used from the design phase. Taking this in account, the width of the channel should be the same all over the way.

The canal must have round edges. A radius of $0,5 \mathrm{~mm}$ until $1 \mathrm{~mm}$ it is necessary for the conception face of the groove. A minimum distance of $0,5 \mathrm{~mm}$ between the material's tongue and the groove is necessary (Figure.3). This will be assured by the alignment of the position of both ends outside the groove's area. Alternating, if this wouldn't be possible, it should exist at least, a small spectrum outside the area. The tolerance must be defined in the consent specifications and between the position limits shown in the below figures.

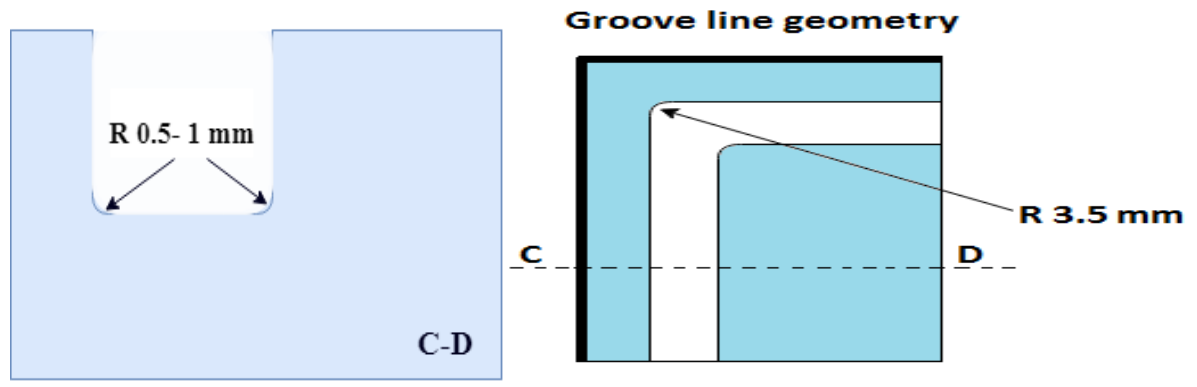

Fig. 3. Dimensions for groove's proposals.

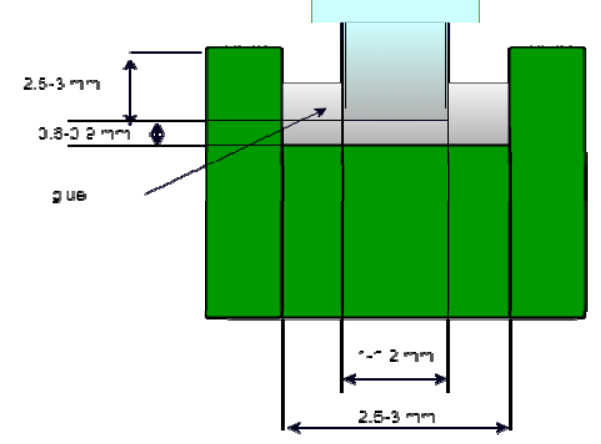

Fig. 4. Dimensions for groove's proposals.

The geometry's groove must be direct proportional with the other counterpart and usually also it should be agreed with production. It should must be considered firstly that the types of connections presented down are not so solid like the upper design. The channel it is composed for this from the radial or axial assembly of the parts (Figure.4.). The glue it is distributed along the groove. For avoiding the leakage outside, the one between the two components assembled must not be bigger than $0,2 \mathrm{~mm}$.

\subsubsection{The sealing by compression}

From the previous experiences in production, it had been determined that for the future application it is better if the material used for gluing must be diffused along one of the parts. Only after this application has been performed, is coming the compression itself. There are two methods tested and that it could work:

- Dry assembly $\rightarrow$ assemblage with a dry and treated material

- Wet assembly $\rightarrow$ assemblage with a humid material

This kind of operations requires that the adhesive to be spread it on the rectangular surface of the working channels, on the design of L surfaces or on plans areas. For the dry 
sealing you need to have the glue only on one side. The flatness of $+/-0.1 \mathrm{~mm}$, to a width of $2 \mathrm{~mm}$ so that the groove can be assured. The design of the leakage surface must assure that it has the space for the gluing to deform. In this way, the groove's dimension have a rate of deformity of minimum $25 \%$. The compression $25-40 \%$ is fine. If is exceeding $40 \%$ or higher, space is the limiting condition and also the fixation is not proper. The sealing material can be pressed up to $50 \%$ (volume-constant medium). This is direct proportional with the deformity's dimensions of the glue. The necessary pressure can be add it by bending or screwing. The distribution of the adhesive must be uniform and that is why a number of adequate fixation points should be also including in the initial designing phase. Like side effect if one of the recommendations was not kept, can appear shrinkage / expansion of the sealing material that is influenced by thermal action [4].

\subsection{Testing's suggestion}

After the designing face, in order to establish if the part has been constructed in a proper way, a series of testing is usually made on the part in general. The suggestion are operations like: temperature step, thermal shocks, salt spray, vibration tests and pressure liquids [4]. The salt spray test it is sustain on approximately 150 hours, on 12 cycles of time, each one consists of 10 hours testing and 5 hours resting at a temperature of $35^{\circ} \mathrm{C}$ with $5 \%$ humidity. The testing that can be done for every part, in serial production, it is the leakage series test. Only performing this tests it is demonstrated why the gluing must possess a specific resistant against the expected flow to inhibit the damage at the sealing surface after numberless hours of assistance [5]. The construction for the beginning of the production's lines and the parts in a proper way will give it a higher amount of energy and productivity keeping the environment not affected [6].

\section{Conclusions}

The silicone and other adhesive materials have been applied from a long time in the past for this operations due to durability and dielectric isolation like barriers against medium contaminations, against shocks and stress vibrations because this materials can absolve them. The items regarding design and suggestions of testing were establish during several mistakes from different projects. The purpose of the research is to have a base for development with the objective of generating designs that are suitable for manufacturing and that can establish in the future a guideline for the process of sealing.

\section{References}

1. S.C Yang, Surface Finishing Theory and New Technology (Machinery Ind.Press, 2000)

2. F. Hashimoto, H. Hamaguchi, CIRP-ANN Manufact, -Technology. 65, 597-620 (2016)

3. T. Siewert, Database for Solder proprieties with emphasis on new lead free solders (2002)

4. K. Fronius, M. Jäckle, B. Bertsche, ATZ Wordwide, 105, 10, 978 (2003)

5. Gh.Nagit, M.I. Ripanu, M. Pista, 112, (EDP Sciences, 2017)

6. L. Tabacaru, D. Suteu, C. Popovici, 15 (EEMJ, 2017) 